\title{
Diferenciais dos indicadores per capita em Manaus: investigação sobre a assimetria entre receita pública e renda
}

\author{
Research on the asymmetry between public revenue and per capita income in \\ Manaus
}

Cleísa Elena Cabral Bessa ${ }^{1 *}$, Bruno de Melo Ruas ${ }^{2}$

\begin{abstract}
RESUMO
A cidade de Manaus vivenciou uma forte expansão econômica, a partir da segunda metade do século XX, em função da criação da Zona Franca de Manaus, modelo de desenvolvimento regional baseado em incentivos fiscais. Apesar de ter experimentado um elevado crescimento econômico, tal situação não implicou na manutenção do nível de receita pública per capita em níveis elevados. Esse diferencial pode estar relacionado a diversos fatores, dentre os quais um baixo grau de esforço fiscal do município de Manaus. 0 presente artigo buscou obter da forma funcional da Receita Potencial, os dados dos níveis do esforço fiscal do município, bem como o levantamento do grau de dependência das transferências governamentias que compõem a receita pública do município, durante os anos de 2002 a 2016. Tais indicadores contribuem na composição de estudos relevantes, inclusive, para auxiliar nas tomadas de decisões realcionadas as políticas públicas, por parte dos gestores muncipais. Os resultados obtidos na presente pesquisa sugerem que durante alguns anos a capacidade de arrecadação do município não foi muito explorada, dado a existência de graus de esforço fiscal abaixo de 1. Além disso, constatou-se que nos anos em que houve alta dependência do ICMS, o município apresentou baixo índice de esforço fiscal, corroborando para o fato de que as transferências intergovernamentais exercem uma influência negativa no esforço de arrecadação municipal.
\end{abstract}

Palavras-chave: Receita Potencial; Política Fiscal; Políticas Públicas.

\begin{abstract}
The city of Manaus experienced a strong economic expansion, from the second half of the 20th century, due to the creation of the Manaus Free Trade Zone, a regional development model based on tax incentives. Despite having experienced high economic growth, this situation did not imply maintaining the level of per capita public revenue at high levels. This differential may be related to several factors, including a low degree of fiscal effort in the municipality of Manaus. The present article sought to obtain from the functional form of the Potential Revenue, the data of the levels of the fiscal effort of the municipality, as well as the survey of the degree of dependence on government transfers that make up the public revenue of the municipality, during the years 2002 to 2016. Such indicators contribute to the composition of relevant studies, including, helping public policy decision making by the municipal managers. The results obtained in the present research suggest that for some years the collection capacity of the municipality was not much explored, given the existence of degrees of fiscal effort below 1. Furthermore, it was found that in the years when there was a high dependence on the ICMS , the municipality presented a low rate of fiscal effort, corroborating the fact that intergovernmental transfers have a negative influence on the municipal collection effort.
\end{abstract}

Keywords: Potencial Revenue; Fiscal Policy; Taxes.

\footnotetext{
${ }^{1}$ Universidade do Estado do Amazonas - UEA. *E-mail: cbessa@uea.edu.br

${ }^{2}$ Bacharel em Ciências Econômicas - UEA
} 


\section{INTRODUÇÃO}

A cidade de Manaus vivenciou crescimento econômico significativo durante o final do século XIX e início do século XX, com o período áureo da extração e exportação da borracha, produto que trouxe grande riqueza a capital amazonense. Após o fim do ciclo de prosperidade, a região passou por quase cinquenta anos de estagnação econômica. Para estimular o crescimento econômico e geração de emprego, o governo Federal criou a Zona Franca de Manaus, modelo de desenvolvimento regional baseado em incentivos fiscais por meio do Decreto-lei 288/1967, cuja finalidade foi a de atrair indústrias e ampliar a geração de emprego e renda. Esse modelo beneficiou economicamente a região por meio da criação de milhares de empregos diretos e indiretos. Consequentemente, a cidade de Manaus se transformou em uma metrópole, com participação relevante na produção na riqueza nacional.

Nos últimos anos do século XX e os primeiros anos do Século XXI, os indicadores de riqueza da cidade de Manaus aprestaram forte expansão, impulsionados pela produção industrial local. Tal desempenho se deu, em grande parte, pela ascensão comercial a partir do modelo Zona Franca de Manaus bem como pelos ganhos de produtividade do trabalho da indústria de transformação amazonense, representada em parte significativa pelo Polo Industrial de Manaus - PIM.

Em relação aos indicadores de desempenho econômico, de acordo com dados do IBGE, em 2012, em relação as capitais, Manaus obteve o sétimo maior PIB per capita, cujo valor foi de $\mathrm{R} \$ 23.286,06$, ficando aproximadamente $38 \%$ acima da média nacional, que era de $\mathrm{R} \$ 16.917,66$.

Na medida em que a atividade produtiva se intensifica, os indicadores conjunturais de desempenho macroeconômico são influenciados, usualmente, no mesmo sentido. Sendo assim, maior produção e vendas implicam em maior arrecadação por parte do setor público. Por exemplo, em cidades como Vitória, São Paulo, Rio de Janeiro, Porto Alegre e Curitiba, que estão entre as cinco maiores capitais em termos de PIB per capita são também as cinco maiores em termos de receita pública per capita.

Entretanto, no caso do município de Manaus, 6a capital em termos de PIB per capita, a receita pública per capita municipal, em 2012, alcançou o valor de R \$ 1.381,61 
(um mil, trezentos e oitenta e um reais e sessenta e um centavos), ocupando a 21a posição dentre as capitais brasileiras.

Diante desta situação, é percebido que um PIB per capita alto não implica necessariamente em receita pública per capita elevada. Tal diferencial pode estar relacionada a diversos fatores como, por exemplo, a política de incentivos fiscais bem como o grau de esforço fiscal do município em questão.

Diante do exposto, a obtenção da forma funcional da Receita Potencial, os dados dos níveis do esforço fiscal do município, bem como o levantamento do grau de dependência das transferências governamentias que compõem a receita pública do município contribuem na composição de estudos relevantes, inclusive, para auxiliar nas tomadas de decisões realcionadas as políticas públicas, por parte dos gestores muncipais.

\section{QUESTÕES FISCAIS: RECEITA PÚBLICA, FEDERALISMO, ESFORÇO E TRANSFERÊNCIAS INTERGOVERNAMENTAIS.}

A operacionalização das atividades e políticas exercidas pelo Estado demandam por recursos oriundos de suas receitas, portanto pesquisas sobre esta temática são significativas não apenas para a compreensão da estrutura das contas públicas, mas também para verificar as possibilidades de elaboração e implementação de políticas públicas (como as de inclusão social) e econômicas (como as fiscais).

Araújo e Arruda (2004) afirmam que receita pública se refere a todo recolhimento de recursos feito aos cofres públicos, representando os valores que o governo tem a receber da arrecadação, de leis, contratos ou de quaisquer outros títulos de que derivem direitos a favor do Estado. A receita pública também pode ser compreendida como a soma de ingressos, impostos, taxas, contribuições e outras fontes de recursos, arrecadados para atender às despesas públicas.

A forma como as fontes de arrecadação governamentais difere no que concerne aos entes da federação. Sendo assim, União, Estados e Municípios apresentam competências e metodologias assimétricas de criação e arrecadação de impostos, taxas e contribuições de melhoria. Tal diferencial é amparado pelo federalismo fiscal.

A Constituição Federal, de 1988 descentralizou para os governos locais grande parte das competências tributárias que anteriormente eram exclusivas da União. Atualmente, os Municípios têm, dentro de seu alcance, o poder e a autonomia de criar determinados tributos, dentro da esfera que lhe e assinalada a Constituição. Segundo 
Amaro (2010), "No que diz respeito às receitas [...], optou a Constituição por um sistema misto de partilha de competência e de partilha do produto da arrecadação. [...] O poder de criar tributos é repartido entre os vários entes políticos."

As competências tributárias são divididas em três tipos: as exclusivas, as imprevistas e as comuns de todos os entes políticos.

\begin{abstract}
"Designa-se privativa a competência para criar impostos atribuída com exclusividade a este ou àquele ente político. [...] Diz-se residual a competência (atribuída à União) atinente aos outros impostos que podem ser instituídos sobre situações não previstas. Seria comum a competência [...] atribuída a todos os entes políticos. [...] A competência tributável é indelegável. Ao destinatário da competência é dado não exercê-la, ou fazê-lo parcialmente [...], mas não lhe é permitido transferir (ou delegar) a competência." (AMARO, 2010, p. 117)
\end{abstract}

Nos últimos anos, o governo federal descentralizou a execução de determinados serviços públicos, principalmente relacionados a área de saúde e educação, que são de competência comum a todos os entes da federação. Tal medida aumentou, significativamente, a responsabilidade dos municípios na condução das políticas públicas. A adoção do modelo de federalismo fiscal implica na partilha da receita tributária e na redistribuição dos recursos entre os entes da federação.

Este procedimento, ou seja, a partilha da receita pública se dá, em grande parte, por meio das transferências intergovernamentais. Em se tratando do alto volume de recursos, são dignos de destaque o Imposto Sobre Circulação de Mercadorias e Serviços - ICMS e o Fundo de Participação dos municípios - FPM.

O ICMS caracteriza-se como uma receita tributária própria dos Estados. De acordo com o Inciso IV do Artigo 158 da Constituição Federal, 25\% de sua arrecadação é repartida aos municípios. No Estado do Amazonas, o ICMS foi regulamentado pela Lei Complementar $n^{\circ}$ 19, de 29 de dezembro de 1997, que instituiu o Código Tributário do Estado do Amazonas.

O valor destinado aos Municípios é repartido de acordo com os coeficientes atribuídos aos mesmos, dentro dos critérios estabelecidos em Lei. Para o ano de 2012, os coeficientes foram definidos pela Portaria n ${ }^{\circ}$ 017/2011 da Secretaria Estadual de Fazenda do Amazonas - SEFAZ/AM e são apresentados no quadro a seguir: 
Quadro 1 - Coeficientes de repartição do ICMS/AM

\begin{tabular}{|c|c|c|c|}
\hline Municípios & Coeficientes & Municípios & Coeficientes \\
\hline ALVARAES & 0,3493180 & JAPURA & 0,4250000 \\
\hline AMATURA & 0,3157180 & JURUA & 0,3585180 \\
\hline ANAMA & 0,3197180 & JUTAI & 0,5223180 \\
\hline ANORI & 0,3557180 & LABREA & 0,7566000 \\
\hline APUI & 0,4178180 & MANACAPURU & 1,3431000 \\
\hline ATALAIA DO NORTE & 0,4983000 & MANAQUIRI & 0,3489180 \\
\hline AUTAZES & 0,5181180 & MANAUS & 62,0936200 \\
\hline BARCELOS & 0,6138000 & MANICORÉ & 0,6662000 \\
\hline BARREIRINHA & 0,3992180 & MARAÃ & 0,3885180 \\
\hline BENJAMIM CONSTANT & 0,5290000 & MAUÉS & 1,1105000 \\
\hline BERURI & 0,3632180 & NHAMUNDÁ & 0,4102180 \\
\hline BOA VISTA DOS RAMOS & 0,3254180 & NOVA OLINDA DO NORTE & 0,4237180 \\
\hline BOCA DO ACRE & 0,5888000 & NOVO AIRÃO & 0,4362180 \\
\hline BORBA & 0,5102180 & NOVO ARIPUANÃ & 0,4162180 \\
\hline CAAPIRANGA & 0,3290180 & PARINTINS & 1,3471000 \\
\hline CANUTAMA & 0,4272180 & PAUINI & 0,4524180 \\
\hline CARAUARI & 0,5288000 & PRES. FIGUEIREDO & 4,2602340 \\
\hline CAREIRO & 0,5246180 & RIO PRETO DA EVA & 0,3810180 \\
\hline CAREIRO DA VARZEA & 0,3846180 & SANTA IZABEL DO RIO NEGRO & 0,4788000 \\
\hline COARI & 2,5552440 & SANTO ANTONIO DO IÇA & 0,4106180 \\
\hline CODAJAS & 0,4823000 & SÃO GABRIEL DA CACHOEIRA & 0,6602000 \\
\hline EIRUNEPE & 0,7039000 & SÃO PAULO DE OLIVENÇA & 0,3977180 \\
\hline ENVIRA & 0,3974180 & SÃO SEBASTIÃO DO UATUMÃ & 0,3267180 \\
\hline FONTE BOA & 0,4086180 & SILVES & 0,3097180 \\
\hline GUAJARA & 0,3512180 & TABATINGA & 0,5907180 \\
\hline HUMAITA & 0,8203000 & TAPAUA & 0,6724000 \\
\hline IPIXUNA & 0,3665180 & TEFE & 0,9915000 \\
\hline IRANDUBA & 0,4345180 & TONANTINS & 0,3407180 \\
\hline ITACOATIARA & 1,9322000 & UARINI & 0,3256180 \\
\hline ITAMARATI & 0,3816180 & URUCARA & 0,5096000 \\
\hline ITAPIRANGA & 0,3260180 & URUCURITUBA & 0,3867180 \\
\hline \multicolumn{3}{|l|}{ TOTAL } & \\
\hline
\end{tabular}

Fonte: SEFAZ/AM (2012) 
Quanto ao Fundo de Participação do Município - FPM, este é composto de 22,5\% dos valores arrecadados através do Imposto de Renda e Impostos sobre Produtos Industrializados, ambos de competência da União. No critério utilizado para o cálculo da distribuição dos recursos aos municípios são considerados o número de habitantes, onde são fixadas faixas populacionais e calculado um coeficiente individual, de acordo com a lei $5.172 / 96$.

Segundo Massardi (2014), as transferências intergovernamentais têm sido utilizadas para reduzir as disparidades socioeconômicas inter-regionais, mas verifica-se também que essas transferências têm desestimulado o esforço de arrecadação própria dos municípios.

Em relação à arrecadação tributária própria, diversos autores ressaltaram a relevância dessa modalidade de receita governamental e a relação com a atividade econômica. Dentre estas pesquisas, são dignas de destaque as de Massardi (2014) e Mendes (2006), que discutiram sobre questões relacionadas ao esforço fiscal nos municípios mineiros e para todos os municípios brasileiros, respectivamente.

Nesses estudos, para que seja discutido o esforço fiscal, foram analisadas variáveis como a receita publica potencial e efetiva, bem como o grau de dependência municipal em relação as transferências intergovernamentais. Estas variáveis podem implicar de forma significativa no processo de desenvolvimento socioeconômico.

Tanto no caso da economia manauara quanto na amazonense, os estudos realizados na temática fiscal, usualmente, têm objetos de estudo concentrados nas questões pertinentes a arrecadação de impostos indiretos como o ICMS, bem como nas implicações e importância deste para o desempenho da economia amazonense como um todo, tal qual foi discutido por Silva (2014), Piancastelli e Perobelli (1996), Paes e Siqueira (2005), dentre outros.

Entretanto, apesar de a arrecadação do ICMS ser parte significativa da receita publica municipal e, consequentemente, contribuir para o orçamento publico, esta não é única variável que compõe as fontes de recursos públicos. Logo, conhecer de forma mais apurada os demais componentes da arrecadação permite um estudo mais aprofundado das questões fiscais.

Portanto, estudos sobre o desempenho destas variáveis frente a indicadores macroeconômicos como o PIB per capita bem como o crescimento populacional e valores 
adicionados setoriais ainda são relativamente escassos. Não obstante, os que tratam do esforço fiscal se restringem ao Estado do Amazonas, como a pesquisa de Carvalho, Oliveira e Carvalho (2007). Sendo assim, estas variáveis, quando analisadas perante a realidade do município de Manaus, podem implicar em informações de importância significativa não apenas para a política fiscal mas também para a melhoria da qualidade de vida da sociedade.

\section{RECEITA POTENCIAL MUNICIPAL, NÍVEL DE ESFORÇO FISCAL E GRAU DE DEPENDÊNCIA.}

Um dos objetivos do presente trabalho refere-se a construção da forma funcional para estimativa da Receita Total do Município de Manaus. Trata-se de um projeto pioneiro, entretanto, conta com um volume de dados significativamente reduzido, em virtude do resultado ser limitado ao horizonte de estudo, de poucos anos.

A versão inicial do modelo possuía uma alta multicolineariedade, o que inviabilizou a utilização simultânea de todas elas no modelo de estimação por mínimos quadrados ordinários; portanto, utilizou-se uma versão reduzida do modelo inicialmente proposto para prosseguimento da pesquisa.

Assim, destaca-se a forma funcional do modelo utilizado para estimativa para Receita Potencial Municipal:

$$
\begin{array}{ll}
\operatorname{Ln}(\mathrm{Rt})=-0,73+0,425 * \operatorname{Ln}\left(\mathrm{PIB} \_\mathrm{REAL}\right)+0,65 * \operatorname{Ln}\left(\mathrm{FPM} \_\mathrm{REAL}\right) & \\
\mathrm{R}^{2}=0,95 & \mathrm{~N}=15
\end{array}
$$

O coeficiente de Pearson é de 95 e amostra de 15. Foram feitos 2 testes de adequação do modelo: Teste reset de Ramsen, Teste de normalidade dos resíduos.

No teste de normalidade dos resíduos, a hipótese nula verificada foi a de normalidade na distribuição do termo erro. A estatística de teste calculada pela distribuição de probabilidade qui-quadrado para 2 graus de liberdade e $5 \%$ de significância foi de 1,68673, cujo valor crítico da rejeição da hipótese nula é de 7,37, ou seja, não rejeitamos a hipótese nula.

O outro teste utilizado foi de detecção de má especificação da forma funcional, $\mathrm{O}$ teste reset de Ramsen. O objetivo desse teste é verificar se a relação formalizada não 
possui não linearidades importantes ausentes. A hipótese nula do teste é a de especificação correta do modelo. A estatística de teste calculada pela distribuição de probabilidade $\mathrm{F}$ foi de 1,26974 para 2 graus de liberdade no numerador e 10 graus de liberdade no denominador, com grau de significância de 5\%, cujo valor crítico calculado é de 5.45, portanto, não se rejeitou-se a hipótese nula do teste.

A utilização da forma funcional para o município de Manaus nos indica que o indicador de Esforço fiscal foi acima de 1, de forma contínua, apenas a partir de 2013. Um índice maior que 1 significa que a receita arrecadada é superior à estimada, consequentemente o município está aproveitando sua base tributária disponível.

O quadro a seguir ilustra o indicador de Esforço Fiscal do município, no período considerado.

Figura 1 - Esforço Fiscal

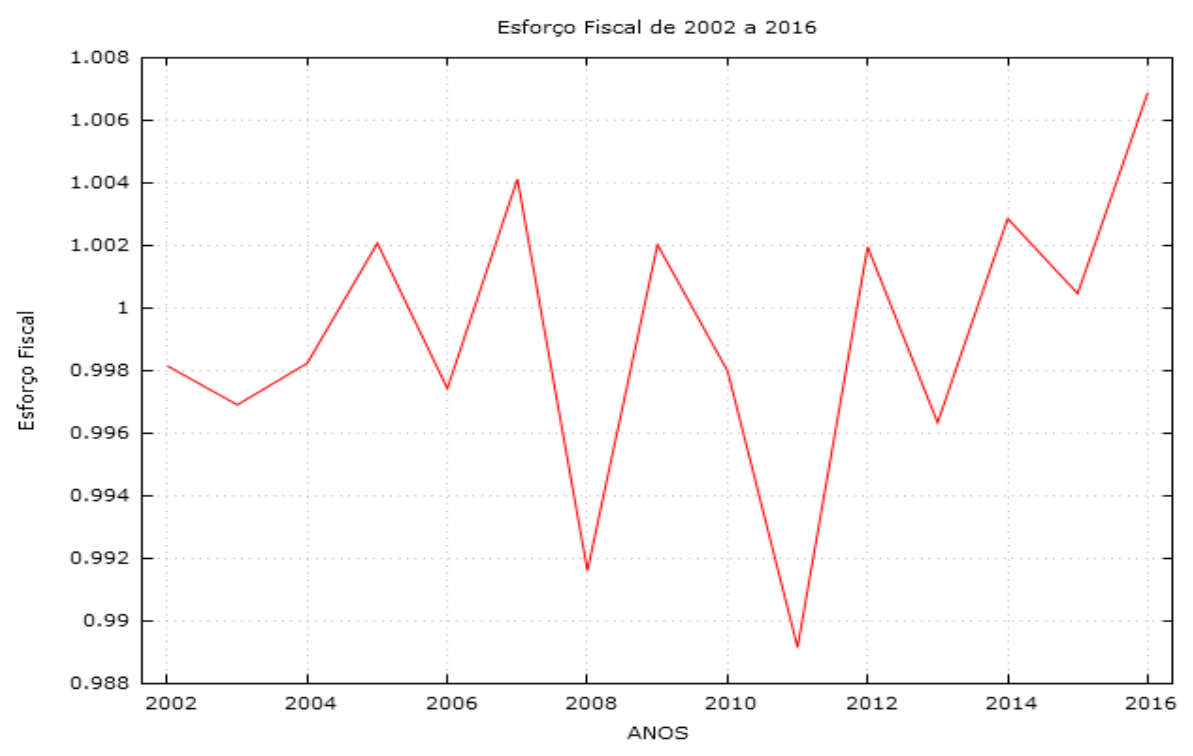

Fonte: Autores.

Em se tratando da análise do nível de dependência da receita publica em relação às transferências governamentais referentes ao repasse do ICMS, constatou-se que o ano de 2014 foi o de maior dependência do período em análise, quando as transferências deste imposto estadual representaram $38,4 \%$ da receita corrente do município. Em relação ao repasse do FPM - Fundo de Participação do Município, o ano de 2016 apresentou mais nível de dependência, quando o FPM totalizou 8,7\% da receita corrente municipal. 2016 foi o ano em que houve o menor nível de dependência do repasse do ICMS, totalizando $27,6 \%$ da receita corrente líquida. Tal redução se fundamenta pela queda de arrecadação 
do estado, em virtude da grave recessão econômica ocorrida, ressaltando que o ICMS é a principal fonte de renda tributária estadual. O ano de 2014 foi o de menor nível de dependência do FPM do período em análise, em um montante de $6,6 \%$ da receita corrente do município.

\section{CONCLUSÃO.}

Em um regime federalista, como o existente no país, coexistem entes subnacionais, autônomos, que devem conciliar um nível ótimo de recursos, de maneira que possam fornecer bens públicos à população local, promovendo a equidade entre as regiões. Nesse sentido, a divisão das competências tributárias e de responsabilidades na prestação de serviços públicos deve ser balanceada e os recursos necessários para financiar tais serviços se originam de tributação própria e transferências intergovernamentais.

A forma como as fontes de arrecadação governamentais difere no que concerne aos entes da federação. Sendo assim, União, Estados e Municípios apresentam competências e metodologias assimétricas de criação e arrecadação de tributos, sua principal fonte de recursos.

A Constituição Federal, de 1988 descentralizou para os governos locais grande parte das competências tributárias que anteriormente eram exclusivas da União. Atualmente, os Municípios têm, dentro de seu alcance, o poder e a autonomia de criar determinados tributos, dentro da esfera que lhe e assinalada a Constituição. Tais competências tributárias são divididas em três tipos: as exclusivas, as imprevistas e as comuns de todos os entes políticos.

Nos últimos anos, o governo federal descentralizou a execução de determinados serviços públicos, principalmente relacionados a área de saúde e educação, que são de competência comum a todos os entes da federação. Tal medida aumentou, significativamente, a responsabilidade dos municípios na condução das políticas públicas.

A adoção do modelo de federalismo fiscal implica na partilha da receita tributária e na redistribuição dos recursos entre os entes da federação. Este procedimento, ou seja, a partilha da receita pública se dá, em grande parte, por meio das transferências intergovernamentais. Em se tratando da área do estudo, são dignos de destaque o Imposto Sobre Circulação de Mercadorias e Serviços - ICMS e o Fundo de Participação dos municípios - FPM. 
O ICMS caracteriza-se como uma receita tributária própria dos Estados. De acordo com o Inciso IV do Artigo 158 da Constituição Federal, 25\% de sua arrecadação é repartida aos municípios. O valor destinado aos Municípios é repartido de acordo com os coeficientes atribuídos aos mesmos, dentro dos critérios estabelecidos em Lei.

Em relação ao Fundo de Participação do Município - FPM, este é composto de 22,5\% dos valores arrecadados através do Imposto de Renda e Impostos sobre Produtos Industrializados, ambos de competência da União.

Neste trabalho, buscou-se analisar o esforço de arrecadação do município de Manaus, no período de 2002 a 2016, bem como a sua relação com o nível de dependência de transferências intergovernamentais, mais especificamente os repasses do ICMS e do FPM.

O índice de esforço fiscal foi obtido por meio da divisão entre a receita tributária efetivamente arrecadada pelo município e a receita potencial estimada por um modelo de regressão. O modelo foi desenvolvido através de variáveis que mensuram a base tributária disponível como o PIB, a população e o nível de atividade industrial, agrícola e de prestação de serviços.

Os resultados obtidos sugerem que durante alguns anos a capacidade de arrecadação do município não foi muito explorada, dado a existência de graus de esforço fiscal abaixo de 1. Além disso, constatou-se que nos anos em que houve alta dependência do ICMS, o município apresentou baixo índice de esforço fiscal, corroborando para o fato de que as transferências intergovernamentais exercem uma influência negativa no esforço de arrecadação municipal.

\section{REFERÊNCIAS}

AMARO, Luciano. Direito Financeiro e Direito Tributário. 16. ed. São Paulo: Saraiva, 2010.

ARAUJO, I; ARRUDA, D. Contabilidade Pública da Teoria a Prática. São Paulo: Saraiva, 2004.

BLANCHARD, Olivier. Macroeconomia. $5^{\text {a }}$ ed. São Paulo: Pearson Prentice Hall, 2011.

BOTELHO, Antônio. Projeto ZFM: Vetor de Interiorização Ampliado. Manaus: s. ed., 2001. 
CARVALHO, David Ferreira; OLIVEIRA, Cléo C. Resque de; CARVALHO, André Cutrim. Desigualdades econômicas inter-regionais, capacidade tributária e esforço fiscal dos estados da Amazônia (1970-2000): uma abordagem econométrica de fronteira estocástica. Novos cadernos NAEA. v. 10, n. 2, p. 5-48, dez. 2007, ISSN $1516-6481$.

GIL, Antônio Carlos. Como elaborar projetos de pesquisa. $5^{\text {a }}$ ed. São Paulo: Editora Atlas, 2010.

MASSASDI, Wellington de Oliveira. Esforço Fiscal e Desempenho Socioeconômico dos Municípios Mineiros. Viçosa: UFV. Dissertação de Mestrado - Universidade Federal de Viçosa, Viçosa, 2014.

McCONNELL, Campbell; BRUE, Stanley. Macroeconomia - Princípios, Problemas e Políticas. 14 ${ }^{\mathrm{a}}$ ed. Rio de Janeiro: LTC, 2001.

MORAES, D. P. Arrecadação tributária municipal: esforço fiscal, transferências e Lei de Responsabilidade Fiscal. São Paulo: FGV, 2006. Dissertação de Mestrado Fundação Getúlio Vargas, São Paulo, 2006.

NEVES, José. Pesquisa Qualitativa - Características, usos e possibilidades. São Paulo: FEA/USP, 1996.

PAES, Nelson Leitão; SIQUEIRA, Marcelo Lettieri. Análise dos Efeitos Econômicos da Implantação do Princípio do Destino na Cobrança do ICMS e suas Implicações sobre a Pobreza e a Desigualdade de Renda. Revista EconomiA. Brasília, v.6, n.3, p.91-126, dez 2005.

PIANCASTELLI, Marcelo; PEROBELLI, Fernando. ICMS: Evolução Recente e Guerra Fiscal. Texto para discussão No 402. Brasília: IPEA, 1996.

SILVA, Sérgio Marinho da. Projeção dos impactos econômicos da redução na alíquota do ICMS na economia do estado do Amazonas: uma abordagem de equilíbrio geral computável (MINIMAN). Dissertação de Mestrado em Economia Pontifícia Universidade Católica do Rio Grande do Sul. Porto Alegre, 2014.

VALLE, Maria Izabel. Globalização e reestruturação produtiva. Um estudo sobre a produção offshore em Manaus. Tese de doutorado. Rio de Janeiro, UFRJ/IFCS, 2000.

VERGARA, Sylvia Constant. Projetos e Relatórios de Pesquisa em Administração. $13^{\circ}$ ed. São Paulo: Atlas. 2011.

Recebido em: 15/09/2021

Aprovado em: 05/10/2021

Publicado em: 10/10/2021 